\title{
DISTÂNCIA DE VOO PARA FORRAGEAMENTO DA ABELHA URUÇU BEIÇO (Melipona eburnea Friese, 1900)
}

\author{
Francisco Cildomar da Silva Correia ${ }^{1}$ \\ Rui Carlos Peruquetti ${ }^{1}$ \\ Andressa Ribeiro da Silva ${ }^{2}$ \\ Fábio Augusto Gomes ${ }^{1}$
}

CORREIA, F. C. da S.; PERUQUETTI, R. C.; SILVA, A. R. da; GOMES, F. A. Distância de voo para forrageamento da abelha Uruçu Beiço (Melipona eburnea Friese, 1900). Arq. Ciênc. Vet. Zool. UNIPAR, Umuarama, v. 20, n. 3, p. 143-146, jul./set. 2017.

RESUMO: Devido à necessidade de insumos para manutenção da colônia, as abelhas operárias realizam voos afim de coletar alimentos (pólen e néctar), materiais de construção (barro, resina etc.) e retirar o lixo do interior da colônia. Para realização do experimento foram capturadas 30 abelhas operárias, na saída do ninho, que foram marcadas no tórax com tinta atóxica e colocadas em uma caixa plástica, medindo $11 \mathrm{~cm}$ de altura por $15 \mathrm{~cm}$ de largura e $20 \mathrm{~cm}$ de comprimento. As cores utilizadas na identificação das abelhas foram administradas de acordo com a distância de soltura. Adotaram-se as cores: laranja para $500 \mathrm{~m}$; azul para $1.000 \mathrm{~m}$; amarela para $1.500 \mathrm{~m}$; verde para $2.000 \mathrm{~m}$; branca para $1.600 \mathrm{~m}$ e vermelha para $1.700 \mathrm{~m}$. As solturas foram realizadas uma a cada dia de experimento, sendo as distâncias medidas com o auxílio de GPS. As liberações ocorreram entre $07 \mathrm{~h}$ e 08h da manhã, em linha reta, em relação à colônia. Para contagem do número de abelhas que retornavam, a entrada do ninho foi fechada e, a cada abelha que regressava, era reaberta, permitindo que a mesma entrasse. Para análise dos dados, utilizou-se o programa Microsoft Excel 2013. Houve uma taxa significativa de retorno até 1.500m. A partir dessa distância, o sucesso das atividades de forrageamento foi inferior a 50\%, tornando-se inviável a realização de coletas, devido à perda de grande número de forrageiras. As análises mostraram que Melipona eburnea alcança um raio de ação em torno de 908 ha, podendo percorrer uma distância de, aproximadamente, $1.700 \mathrm{~m}$ em relação ao ninho.

PALAVRAS-CHAVE: Atividade de forrageamento. Raio de ação. Soltura.

\section{FORAGING FLIGHT DISTANCE FOR Melipona eburnea Friese BEES}

\begin{abstract}
Due to the need for inputs to maintain the colony, worker bees fly to collect food (pollen and nectar), building material (clay, resin, etc.) and remove garbage from the inside of the hive. In order to carry out this experiment, a total of 30 foraging bees were collected at the exit of the hive, marked on the chest with nontoxic paint and placed in a plastic box measuring $11-\mathrm{cm}$ high, $15-\mathrm{cm}$ wide and $20-\mathrm{cm}$ long. The colors used for the identification of the bees were administered according to the distance of their release. The following colors were adopted: orange for $500 \mathrm{~m}$; blue for 1,000 m; yellow for $1,500 \mathrm{~m}$; green for 2,000 m; white for 1,600 m, and red for 1,700 m. The releases were performed one per day of the experiment, and the distances measured with the aid of a GPS device. The releases took place between 7 and 8 a.m. in a straight line in relation to the hive. In order to count the number of returning bees, the nest entrance was closed and only reopened for each returning bee, allowing it to enter. Data was analyzed used Microsoft Excel 2013. There was a significant rate of return up to $1,500 \mathrm{~m}$. Beyond that distance, the success of foraging activities was less than $50 \%$, making data collection impossible due to the loss of large number of worker bees. The analyzes showed that Melipona eburnea reaches an action radius of around 908 ha, being able to cover a distance of approximately $1,700 \mathrm{~m}$ in relation to the hive.
\end{abstract}

KEYWORDS: Action radius. Foraging activity. Release.

DISTANCIA DE VUELO PARA FORRAJEO DE ABEJA REAL (Melipona eburnea Friese, 1900)

RESUMEN: Debido a la necesidad de insumos para el mantenimiento de la colonia, las abejas obreras realizan vuelos para recolectar alimentos (polen y néctar), materiales de construcción (arcilla, resina, etc.) y quitar la basura desde el interior de la colonia. Para realizar el experimento fueron capturados 30 obreras a la salida del nido, que estaban marcadas en el pecho con tinta no tóxica y puestas en una caja de plástico, midiendo $11 \mathrm{~cm}$ de alto, $15 \mathrm{~cm}$ de ancho y $20 \mathrm{~cm}$ de longitud. Los colores utilizados en la identificación de las abejas fueron manejados de acuerdo con la distancia de soltura. Se adoptaron los colores: naranja de $500 \mathrm{~m}$; azul para $1.000 \mathrm{~m}$; amarillo para $1.500 \mathrm{~m}$; verde para $2.000 \mathrm{~m}$; blanco para $1.600 \mathrm{~m}$ y rojo para $1.700 \mathrm{~m}$. Las solturas se realizaron una a cada día del experimento, y las distancias medidas con la ayuda de un GPS. Las liberaciones ocurrieron entre las $07 \mathrm{~h}$ y $08 \mathrm{~h}$ de la mañana, en línea recta, en relación con la colonia. Para conteo del número de abejas que retornaban, la entrada del nido fue cerrada y, a cada abeja que regresaba era reabierta, permitiendo que la misma entrase. Para análisis de los datos se utilizó el programa Microsoft Excel 2013. Hubo una tasa significativa de retorno hasta 1.500m. A partir de esta distancia, el éxito de las actividades de forrajeo fue inferior al 50\%, lo que hace inviable la realización de recolecciones, debido a la pérdida de un gran número de forrajes. El análisis mostró que Melipona eburnea alcanza un radio de acción alrededor de 908ha, pudiendo recorrer una distancia de, aproximadamente, 1.700m en relación al nido.

DOI: 10.25110 /arqvet.v20i3.2017.5838

${ }^{1}$ Professor da Universidade Federal do Acre - UFAC. Programa de Pós-Graduação em Sanidade e Produção Animal Sustentável na Amazônia Ocidental. ${ }^{2}$ Graduanda em Medicina Veterinária. Bolsista PIBIC. Universidade Federal do Acre - UFAC. 
PALABRAS CLAVE: Actividad de forrajeo. Radio de acción. Soltura.

\section{Introdução}

As abelhas da subtribo Meliponina possuem ferrão atrofiado, são eussociais e vivem em colônias perenes (MICHENER, 1979), cuja população pode variar de poucas centenas a dezenas de milhares de indivíduos (SOMMEIJER, 1999; CAMPOS, 2003). A estrutura e localização dos ninhos das abelhas sem ferrão variam de acordo com a espécie (CAMPOS.; PERUQUETTI, 1999), podendo apresentar heterogeneidade quanto à cor, tamanho, forma, hábitos de nidificação e tamanho da população de seus ninhos, sendo que muitas podem ser criadas racionalmente (PEREIRA, 2005).

Devido à necessidade de insumos para manutenção da colônia, as abelhas operárias realizam voos afim de coletar alimentos (pólen e néctar), materiais de construção (barro, resina, etc.) e para retirar o lixo da colônia (NOGUEIRA-NETO, 1997; TEIXEIRA; CAMPOS, 2005). A distância percorrida pelas operárias durante suas atividades de forrageamento depende de fatores, como: densidade, sazonalidade da fonte de alimento, tamanho do corpo da abelha (DORNHAUS et al., 2006), bem como condições da colônia e fatores climáticos, principalmente a temperatura (ROUBIK, 1989; HILÁRIO, et al., 2001). Todavia, para aumentar a eficiência na exploração de recursos, as abelhas eussociais desenvolveram mecanismos sofisticados de comunicação entre elas, os quais permitem o recrutamento de outras abelhas para um local com abundância de alimentos (NIEH, 2004; AGUILAR et al., 2005). Além disso, as abelhas não forrageiam em longas distâncias desnecessariamente, poupando assim, tempo e energia (FRISCH, 1967; SEELEY, 1994). Análises realizadas com abelhas do gênero Melipona, (Melipona bicolor; Melipna scutellaris e Melipona mandacaia) tiveram suas distâncias máximas de voo estabelecidas em $2.000 \mathrm{~m}$ e $2.100 \mathrm{~m}$, respectivamente (ARAÚJO, et al., 2004; KUHN-NETO, et al., 2009). Já para as espécies Trigona corvina, Partamona aff. cupira, Tetragonisca angustula e Nannotrigona testaceicornis perilampoides, suas distâncias máximas de voo foram estimadas em 623 a 853m (VAN NIEUWSTADT.; RUANO IRAHETA, 1996). No entanto, esse tipo de estudo tem sido realizado com poucas espécies de abelhas sem ferrão.

Para a espécie Melipona eburnea, até o momento, não existem levantamentos que demonstrem a sua distância máxima de voo. Nesse sentido, o presente estudo teve como objetivo compreender os limites percorridos por essa espécie em suas atividades de forrageamento, considerando que $M$. eburnea está entre as espécies de abelhas sem ferrão mais criadas racionalmente, entre os estados do Acre e Amazonas (MAGALHÃES.; VETURUERI, 2010). Sendo assim, conhecer o tamanho da área explorada por M. eburnea pode fornecer ao meliponicultor informações relacionadas ao raio de ação de maior atividade dessas abelhas em relação aos recursos alimentares disponíveis em sua propriedade, aumentando as possibilidades de crescimento da produção de mel.

\section{Material e Métodos}

O estudo foi conduzido entre os dias 06 a 21 de março de 2016, em um meliponário localizado a $3 \mathrm{Km}$ de Rio Branco-Acre, entre as coordenadas (9॰55'56" S e 6753'19"
W), a uma altitude é de aproximadamente $148 \mathrm{~m}$. Uma colônia foi selecionada para realização do experimento. Foram capturadas com rede entomológica 30 abelhas operárias, na saída do ninho, às $06 \mathrm{~h}$ da manhã, a cada dia de realização do experimento. As mesmas foram marcadas no tórax com tinta atóxica e colocadas em uma caixa plástica, medindo $11 \mathrm{~cm}$ de altura por $15 \mathrm{~cm}$ de largura e $20 \mathrm{~cm}$ de comprimento, com tampa transparente e furos nas laterais para circulação de ar, contendo um recipiente com algodão, embebido em mel de Apis mellifera, servindo como alimentador.

As cores utilizadas na identificação das abelhas foram administradas de acordo com a distância de soltura, sendo adotadas as cores: laranja para $500 \mathrm{~m}$; azul para $1.000 \mathrm{~m}$; amarela para $1.500 \mathrm{~m}$; verde para $2.000 \mathrm{~m}$; branca para $1.600 \mathrm{~m}$ e vermelha para $1.700 \mathrm{~m}$. As seis solturas foram realizadas uma a cada dia de experimento, sendo as distâncias medidas com o auxílio de GPS.

As liberações ocorreram entre $07 \mathrm{~h}$ e $08 \mathrm{~h}$ da manhã, em linha reta em relação à colônia. Para contagem do número de abelhas que retonavam, a entrada do ninho foi fechada e, a cada abelha que regressava, era aberta, permitindo que a mesma entrasse e, após, era fechada novamente. O total de abelhas que voltaram ao ninho foi anotado para posterior cálculo de porcentagem de sucesso de retorno, juntamente com os dados climáticos do período do experimento. Para análise dos dados utilizou-se o programa Microsoft Excel 2013.

\section{Resultados e discussão}

Foi liberado um total de 180 abelhas pelo método de captura e recaptura de Roubik e Aluja (1983), durante o período de realização do estudo, assumindo que as abelhas que retornassem ao ninho, conheciam o caminho de volta, ou seja, já tinham voado as distâncias utilizadas no experimento. Para Silva et al. (2014), o teste de captura e recaptura pode ser considerado eficaz, uma vez que as abelhas libertadas só precisam voar de volta para o ninho, entretanto, quando realizado em grandes distâncias as abelhas podem se perder no caminho de volta, por falta de familiaridade com o local de onde foram liberadas.

Com relação à distância de soltura das abelhas, iniciou-se a $500 \mathrm{~m}$ do ninho, com temperatura de $26^{\circ} \mathrm{C}$; umidade relativa de $94 \%$ e velocidade do vento de $2 \mathrm{~m} / \mathrm{s}$, com sucesso de retorno de $100 \%$. Posteriormente, o experimento foi repetido, considerando as seguintes distâncias: $1.000 \mathrm{~m}$, com temperatura de $24^{\circ} \mathrm{C}$; umidade relativa de $98 \%$ e velocidade do vento de $4 \mathrm{~m} / \mathrm{s}$, com sucesso de retorno de $86,6 \% ; 1.500 \mathrm{~m}$, com temperatura de $24^{\circ} \mathrm{C}$; umidade relativa de $94 \%$ e velocidade do vento de $2 \mathrm{~m} / \mathrm{s}$, com sucesso de retorno de $63,3 \%$; e $2.000 \mathrm{~m}$, com temperatura de $24^{\circ} \mathrm{C}$; umidade relativa de $98 \%$ e velocidade do vento de $4 \mathrm{~m} / \mathrm{s}$, com sucesso de retorno de $0 \%$. Como não houve retorno das abelhas a $2.000 \mathrm{~m}$, as solturas passaram a ser realizadas a cada $100 \mathrm{~m}$, partindo dos $1.500 \mathrm{~m}$. A $1.600 \mathrm{~m}$, a temperatura era de $26,8^{\circ} \mathrm{C}$; umidade relativa de $98 \%$ e velocidade do vento de $2 \mathrm{~m} / \mathrm{s}$, com sucesso de retorno de $46,6 \%$; a $1.700 \mathrm{~m}$, com temperatura de $24,6^{\circ} \mathrm{C}$; umidade relativa de $98 \%$ e velocidade do vento de $2 \mathrm{~m} / \mathrm{s}$, com sucesso de retorno de $43 \%$. A porcentagem de sucesso para o retorno ao ninho diminuiu gradativamente à medida que as distâncias foram aumentadas (Figura 1). 
Figura 1: Proporção de operárias soltas que retornaram ao seu ninho, a partir de diferentes distâncias. Valor sem retorno de operárias foi omitido da análise.

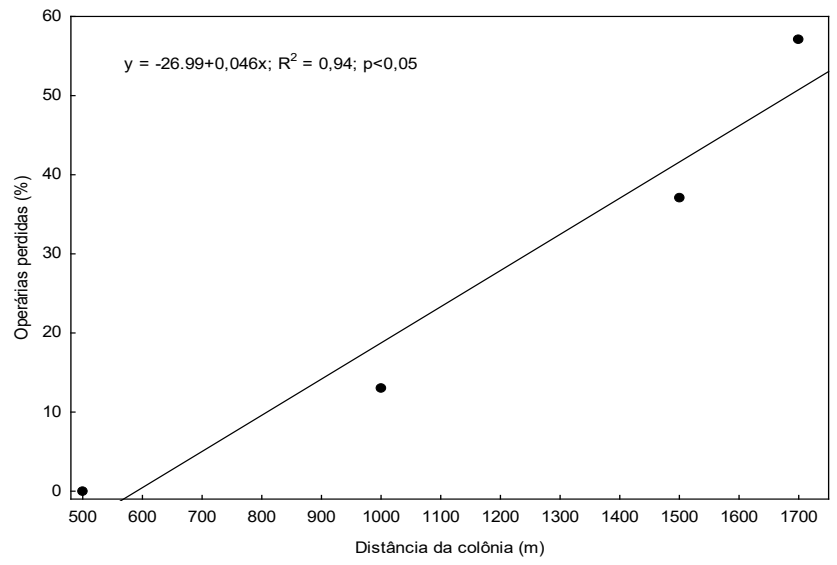

Isso pode ter relação com a preferência das abelhas em forragearem próximas ao ninho, pois segundo Pierrot e Schlindwein, (2003) e Ferreira (2008), com essa prática, a abelha gasta menos tempo em cada coleta, faz menos esforço e transporta maior quantidade de recursos. Dessa forma, tem maior facilidade para o recrutamento de forrageiras e, consequentemente, sucesso no forrageamento e maior probabilidade no processo de polinização das plantas (VAN NIEUWSTADT.; IRAHETA, 1996). Considerando uma provável distância para o recrutamento de operárias, nosso estudo aponta que isso pode ocorrer a cerca de $500 \mathrm{~m}$ do ninho.

Contudo, houve uma taxa significativa de retorno até $1.500 \mathrm{~m}$ (Figura 1). A partir dessa distância, a taxa de sucesso nas atividades de forrageamento são inferiores a $50 \%$, tornando inviável a realização de coletas, devido à perda de grande número de forrageiras. No entanto, M. eburnea se distanciando até $1.700 \mathrm{~m}$ do ninho, é capaz de coletar recursos em uma área de aproximadamente 908 ha como pode ser observado na figura 2 .

Figura 2: Área explorada por Melipona eburnea para coleta de recursos florais. Círculo amarelo: área potencial de forrageamento $(907,92 \mathrm{ha})$; linha verde: distância máxima estimada de voo $(1700 \mathrm{~m})$. Linhas vermelhas: áreas potencialmente exploradas (355,7ha). Imagem datada de 29/06/2016.

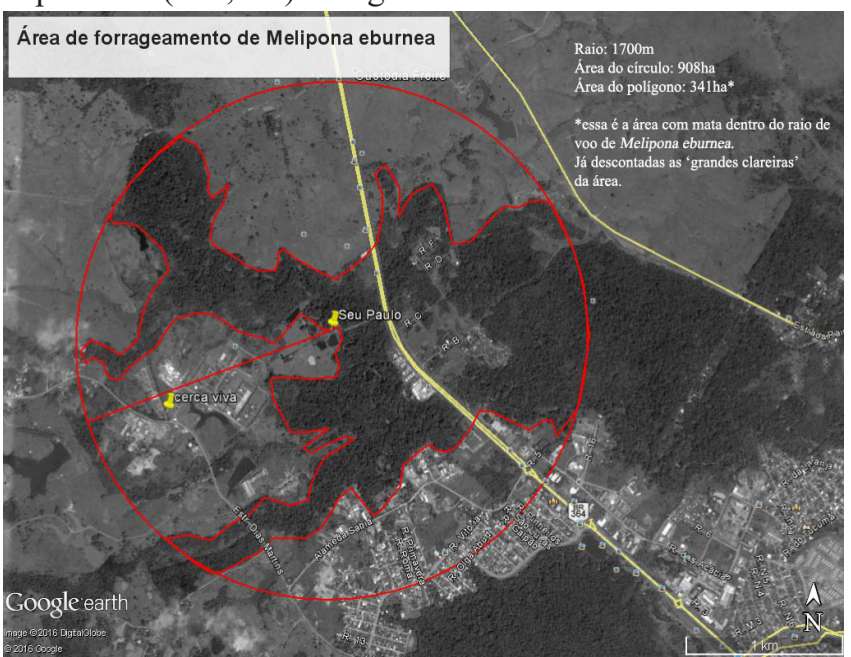

Para Dornhaus et al. (2006), as distâncias percorridas pelas abelhas operárias para o forrageamento depende de inúmeros fatores, dentre eles, a densidade e a sazonalidade da fonte de alimento, a espécie de abelha a e sua fisiologia e, em especial, a correlação entre o tamanho de seu corpo e de suas asas (CASEY, et al., 1985; BYRNE, et al., 1988).

Para Roubik e Ackerman (1987); Baumgartner e Roubik (1989) o tamanho do corpo das operárias é considerado como uma adaptação para a atividade de forrageamento e exploração floral de recurso, sendo que $75,5 \%$ da variação do tamanho do corpo dos meliponíneos correspondem a fatores adaptativos, associados à exploração de recursos (PIGNATA.; DINIZ-FILHO, 1996). Para Araújo et al. (2004), mais de $84 \%$ da distância máxima de voo percorrida pelas abelhas pode estar relacionado com o tamanho de suas asas. Além disso, fatores como condições internas à colônia e fatores climáticos, separados ou em conjunto, podem afetar as atividades de voo das abelhas (HILÁRIO, et al., 2001).

Os resultados do presente trabalho sugerem que a distância percorrida por M. eburnea para coleta de recursos é de aproximadamente $1.700 \mathrm{~m}$. Este valor foi inferior aos relatados por Kerr (1987) para Melipona fasciculata - 2.470m; Rodrigues (2012) para Melipona mandacaia $-2.700 \mathrm{~m}$ e superiores aos resultados de Silva et al. (2014) para Melipona subnitida $-1.160 \mathrm{~m}$.

\section{Conclusão}

Com base nos resultados, as análises mostraram que M. eburnea alcança um raio de ação em torno de 908ha, podendo percorrer uma distância de aproximadamente $1.700 \mathrm{~m}$ em relação ao ninho. Dessa forma, o presente estudo pode ser útil em programas de polinização em áreas cultivadas no raio de voo dessa espécie de abelha, resultando em maior produtividade das culturas. Além disso, conhecer a área efetiva de forrageamento das operárias de $M$. eburnea facilita a formação de pastos meliponícolas adequados à espécie, a fim de garantir o desenvolvimento das colônias, bem como maior produção de mel.

\section{Referências}

AGUILAR, I.; FONSECA, A.; BIESMEIJER, J. C. Recruitment and communication of food source location in three species of stingless bees (Hymenoptera, Apidae, Meliponini). Apidologie, v. 36, n. 3, p. 313-324, 2005.

ARAUJO, E. D. et al. Body size and flight distance in stingless bees (Hymenoptera: Meliponini): Inference of flight range and possible ecological implications. Brazilian Journal of Biology, v. 64, n. 3b, p. 563-568, 2004.

BAUMGARTNER, D. L.; ROUBIK, D. W. Ecology of necrophilous and filth-gathering stingless bees (Apidae: Meliponinae) of Peru. Journal of the Kansas Entomological Society, v. 62, n. 1, p. 11-22, 1989.

BYRNE, D. N.; BUCHMANN, S. L.; SPANGLER, H. G. Relationship between wing loading, wingbeat frequency and body mass in homopterous insects. Journal of Experimental Biology, v. 135, p. 9-23, 1988.

CAMPOS, L. A. O. A criação de abelhas indígenas sem ferrão. Viçosa: Universidade Federal de Viçosa, 2003. 8 p. 
(Informe Técnico, 67).

CAMPOS, L. A. O.; PERUQUETTI, R. C. Biologia e criação de abelhas sem ferrão. Viçosa: Universidade Federal de Viçosa, 1999. 36 p.

CASEY, T. M.; MAY, M. L.; MORGAN, K. R. Flights energetics of euglossinae bees in relation to morphology and wing stroke frequency. Journal of Experimental Biology, v. 116, p. 271-289, 1985.

DORNHAUS, A. et al. Benefits of recruitment in honey bees: effects of ecology and colony size in an individual based model. Behavioral Ecology Journal, v. 17, n. 3, p. 336-344, 2006.

FERREIRA, M. G. Pólen coletado por Scaptotrigona depilis (Moure, 1942) (Hymenoptera: Meliponina), na região de Dourados-MS. Dourados, 2008. 39 f. Dissertação (Mestrado em Entomologia) - Universidade Federal da Grande Dourados, Dourados, 2008.

FRISCH, K. VON. The dance language and orientation of bees. Cambridge: Harvard University Press, 1967. 592 p.

HILÁRIO, S. D.; IMPERATRIZ-FONSECA, V. L.; KLEINERT, A. G. Responses to climatic factors by foragers of Plebeia pugnax Moure (in litt) (Apidae, Meliponinae). Revista Brasileira de Biologia, v. 61, n. 2, p. 191-196, 2001.

KERR, W. E. Biologia, manejo e genética de Melipona fasciculata Smith (Hymenoptera Apidae). Universidade Federal do Maranhão, São Luiz, 1987. 141 p.

KUHN-NETO, B. et al. Long distance foraging and recruitment by a stingless bee, Melipona mandacaia. Apidologie, v. 40, n. 4, p. 472-480, 2009.

MAGAlHÃES, T. L.; VENTURIERI, G. C. Aspectos econômicos da criação de abelhas indígenas sem ferrão (Apidae: Meliponini) no nordeste paraense. Belém:

Embrapa Amazônia Oriental, 36 p. Documentos 364, 2010.

MICHENER, C. D. Biogeography of the bees. Annals of the Missouri Botanical Garden, v. 66, n. 3. p. 277-347, 1979.

NIEH, J. C. Recruitment communication in stingless bees (Hymenoptera, Apidae, Meliponini). Apidologie, v. 35, n. 2, p. 159-182, 2004.

NOGUEIRA-NETO, P. Vida e criação de abelhas sem ferrão. São Paulo: Nogueirapis, 1997. 445 p.

PIERROT, L. M.; SCHLINDWEIN, C. Variation in daily flight activity and foraging patterns in colonies of uruçu Melipona scutellaris Latreille (Apidae, Meliponini). Revista Brasileira de Zoologia, v. 20, n. 4, p. 565-571, 2003.

PIGNATA, M. I. B.; DINIZ-FILHO, J. A. F. Phylogenetic autocorrelation and evolutionary constraints in worker body size of some neotropical stingless bees (Hymenoptera, Apidae). Heredity, v. 76, n. 3, p. 222-228, 1996.

RODRIGUES, F. Aspectos do voo de Melipona mandacaia (Hymenoptera, Apidae, Meliponini) na região do Vale do Submédio São Francisco. 2012. $81 \mathrm{f}$. Dissertação (Mestrado em Ciência Animal) - Universidade Federal do Vale do São Francisco, Petrolina, 2012.

ROUBIK, D. W.; ACKERMAN, J. D. Long-term ecology of euglossine orchid-bees in Panamá. Oecologia, v. 73, n. 3, p. 321-333, 1987.

ROUBIK, D. W. Ecology and natural history of tropical bees. Cambridge University Press. Cambridge, 1989. 514 p.

SEELEY, T. D. Honey bee foragers as sensory units of their colonies. Behavioral Ecology and Sociobiology, v. 34, n 1, 51-62, 1994.

SILVA, A. G. et al. Foraging distance of melipona subnitida Ducke (Hymenoptera: Apidae). Sociobiology, v. 61, n. 4, p. 494-501, 2014

SOMMEIJER, M. J. Beekeepping with stingless bees: a new type of hive. Bee World, v. 80, n. 2, p. 70-79, 1999.

TEIXEIRA, L. V.; CAMPOS, F. N. M. Início da atividade de voo em abelhas sem ferrão (Hymenoptera, Apidae): influência do tamanho da abelha e da temperatura ambiente. Zoociências Juiz de Fora, v. 7, n. 2, p. 195-202, 2005.

VAN NIEUWSTADT, M. G. L.; IRAHETA, C. E. R. Relation between size and foraging range in stingless bees (Apidae, Meliponinae). Apidologie, v. 27, n. 4, p. 219-228, 1996.

Recebido em: 31.08.2016. Aceito em: 09.10.2017. 